\title{
EXPERIMENTAL INVESTIGATION OF THE EFFECT OF THE INTERNAL TWO-PHASE FLOW AND THE BUBBLE SIZE ON THE EFFERVESCENT SPRAY CHARACTERISTICS
}

\author{
Farid A. Hammad ${ }^{1}$, Aly H. Gadallah ${ }^{1}$, E. A. El-Shenawy ${ }^{1}$, Zakarya A. Zyada ${ }^{1,2}$ \\ ${ }^{l}$ Mech. Power Eng. Dep., Faculty of Eng., Tanta University, Egypt \\ ${ }^{2}$ Faculty of Mech. Eng., Universiti Teknologi Malaysia, Johor Bahru, Malaysia \\ farid_hammad@f-eng.tanta.edu.eg,alyhafezg@gmail.com, shenawy49@yahoo.com, \\ zzyada@f-eng.tanta.edu.eg
}

\begin{abstract}
Reducing the pressurized atomizing gas in the effervescent atomizers with keeping fine spray is a desirable aim. This experimental study focuses on improving the characteristics of the effervescent spray (i.e. drop size and spray cone angle); while an "in-out" gas injection configuration atomizer operates under low gas-to-liquid ratios (GLR). These important characteristics are mainly affected by the internal two-phase flow pattern. Wherefore, shadowgraphy is utilized for visualizing and investigating the internal two-phase flow pattern directly upstream the exit orifice. The atomizer is operated at air gauge pressure of $0.6 \mathrm{Mpa}$ and GLRs of $(0.71 \%-5.74 \%)$. For controlling the internal flow at the operating GLRs, two novel bubble breakers (BB) are designed and tested. The bubble breakers are fixed in the mixing chamber to affect the bubble size. The shadowgraph technique is utilized for visualizing and quantifying bubbles inside the atomizer, spray characteristics and the near nozzle spray. From the visualization and results, the internal two-phase flow patterns inside the mixing chamber vary from bubbly flow at the lowest three GLRs to a bubble-annular flow at the largest GLR, passing through an unsteady transition stage between these two patterns. Also, it is observed that the bubble breakers efficiently disintegrate large bubbles; the largest reduction percentage in the bubble size is $75.63 \%$ for the case of flat end BB at GLR of $2.21 \%$. Also, the results showed that the drop size and spray cone angle are strongly affected by the bubble size (i.e. the internal flow structure) subsequently the presence of the bubble breakers. The largest reduction percentage in the droplet size is $68.88 \%$ for the case of the cone end BB at GLR of $0.77 \%$ and the largest increment percentage in the spray cone angle is $69.97 \%$ for the cone end BB at GLR of $0.77 \%$, all relative to the case of without BB.

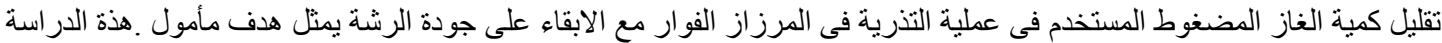

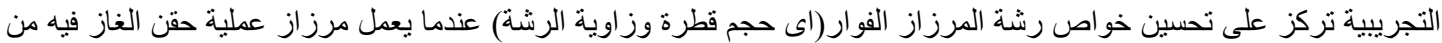

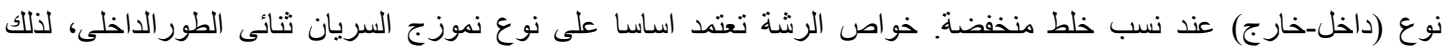

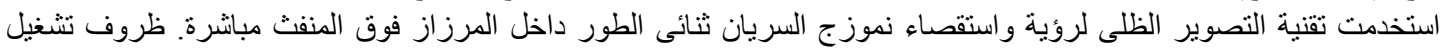

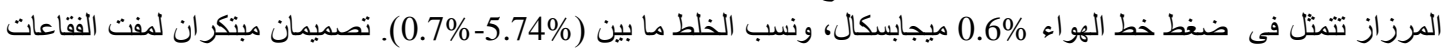

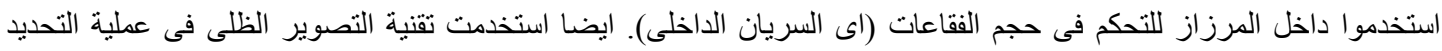

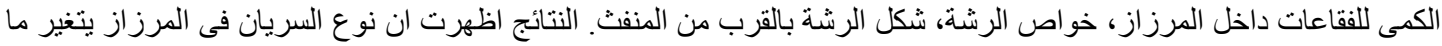

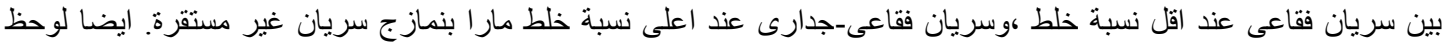




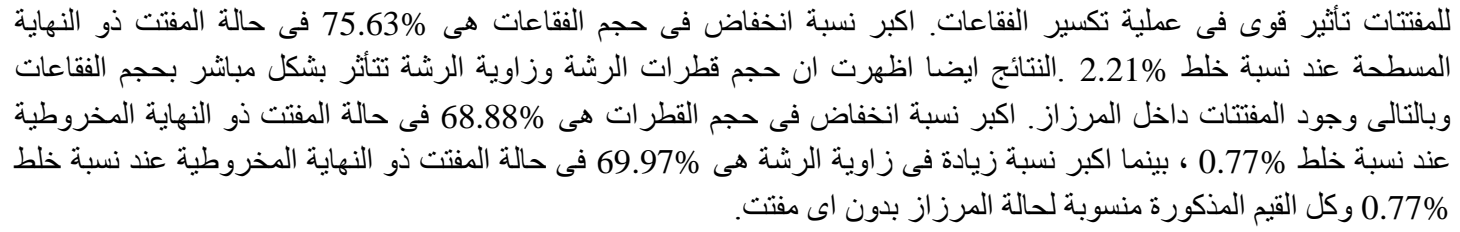

Keywords : effervescent spray; bubble breaker; two-phase flow pattern; shadowgrahpy; bubble size; bubble fragmentation.

\section{INTRODUCTION}

Protect the environment from pollution means safeguard our health from diseases. Environment pollution is a global problem which has a great influence on the human health [1]. Combustion processes are one of the major sources of this pollution [2]. These combustion processes are indispensable in numerous applications. So, we must improve these processes to control and reduce the exhaust pollutant emissions. The performance of combustion process (subsequently the application efficiency) mainly depends on the fuel injection (i.e. liquid fuel atomization) process [3]-[5]. Atomization is the process of converting a bulk liquid to discrete droplets in gas (i.e. spray) [6]-[8]. Devices used to produce sprays are defined as atomizers or spray nozzles[6]. Many types of atomizers (i.e. spray types) have been designed to meet the applications requirements. In the combustion processes, the high evaporation rate of spray droplets is a crucial step for achieving a complete combustion. This requires generating sprays with very fine droplets that have large surface area to enhance evaporation rate [4][6]. One of the most efficient types of atomizers recently utilized in combustion applications is the "effervescent atomizers" [3]-[10].

Effervescent atomizers are a special form of the twin-fluid atomizers, they are internal mixing type, in which the bulk liquid is aerated by a small amount of gas forming bubbly two-phase flow inside the mixing chamber upstream the exit orifice of the atomizer. At the instant that this bubbly mixture exits the discharge orifice, the pressure of the liquid sharply drops causing the gas bubbles expand rapidly, consequently disintegrating the liquid into droplets [3][8][11]-[13].

Many advantages for the effervescent atomizers over the conventional atomizers, comprising lower injection pressures, fine sprays obtained at any injection pressure, consuming lower gas flow rates and larger discharge orifices. The effervescent atomizers can deal with wide range of liquids due to its insensitivity to the liquid physical properties, simple design and nearly no maintenance requirements [3], produce low pollutant emissions when used in combustion systems due to the presence of air (atomizing gas) in the spray core [3]-[11]. These advantages enabled the effervescent atomizers to be utilized in many applications, including gas turbine combustors [12]-[14], scramjet engines [4], IC engines [15]-[17], furnaces and boilers [18]-[20], also used in humidification, dust control, gas cooling, spray coating processes [8], spray drying (or spray crystallization) in food industry [21][22], pharmaceutical, and consumer products [23].

Many parameters affect the effervescent atomizer produced spray. It was observed that the internal flow pattern greatly affects the external flow (i.e. spray) [21][24]-[26]. The internal flow pattern can be evaluated using theoretical or experimental flow regime maps. Researchers [27][28] visualized the actual internal flow and compared it with the predicted flow regime of the maps; some differences between the observed and predicted were noted. It was observed that the two-phase mixing mechanism affects the internal flow inside these atomizers and it was concluded that estimating the flow regime using maps only was not suitable [29]. The internal flow inside the atomizer has been classified into four regimes bubbly, plug, slug, and annular flow [27]-[29]. With increasing the gas to liquid ratio (GLR) the flow regime changes from bubbly to annular flow [27][28][30]. The annular flow produces fine (small drop size) sprays compared 
to the other regimes, though it needs very high gas flow rate [3][30]. The atomizing gas is the key in the effervescent atomization mechanism; high rates of gas enhance the atomization that may be not available in some applications, so we must deal with bubbly flow to produce fine spray. Bubble size has a strong effect on the droplet size [9]. Many mechanisms are used to produce and break up bubbles inside the atomizers. Sutherland et al. [31] used a perforated sheet at a GLR of 0.02 leading to smaller droplet size. Gomez et al. [9] experimentally used two metal plates with small thickness and different holes size in the mixing chamber which disintegrate large bubbles into small ones, thereby producing small drop sizes. They also noted that there was an improvement in the atomizer performance with the presence of the bubble breaker plates compared to its absence at given GLRs. Jagannathan et al. [32] experimentally investigated the effect of $20 \mathrm{kHz}$ ultrasound on the bubbles breakup inside the mixing chamber of a horizontal effervescent atomizer. They found that large bubble was disintegrated to finer ones. They also noted finer spray was produced at GLR of $0.063 \%$ in the presence of ultrasound. But ultrasound power was consumed for this breaking process.

In this study, for the aim of reducing the power consumed for atomization in the form of high gas flow rates, we focused on study and improve the atomizer performance while operating at low GLRs, visualizing the internal flow and detect its pattern. The influence of the novel designs of acrylic bubble breakers on the internal two-phase flow pattern, the near nozzle spray structure, spray cone angle and spray drop size are investigated and comparing them to the case of without BB.

\section{EXPERIMENTAL SETUP AND TECHNIQUES}

\subsection{Test Rig [Fluid Supply System]}

A schematic drawing and photographs of the test rig used in this study are shown in Fig. 1. In this study, the liquid and gas phases are water and air, respectively. Beginning with the plunger pump which takes the water from the funnel and the output discharge is controlled by a bypass line to the funnel. Part of this discharge is directed toward the atomizer, passing through a non-return valve, then to an accumulator used for (reducing) damping discharge (flow) fluctuation, then to a needle valve for adjusting the flow, then to a rotameter used for measuring its volume flow rate with an accuracy of $\pm 4 \%$, then to a pressure gage to measure its pressure, finally to the atomizer. The pressurized air comes from large tank connected to double stage compressor. This air passes through gate valve, pressure regulator for regulating the air pressure, then to needle valve, a rotameter for measuring the air volume flow rate with an accuracy of $\pm 5 \%$, pressure gauge to measure its pressure, then to non-return valve for safety of the back flow of water to the air line, finally to the atomizer. The funnel is used to collect the spray.

\subsection{Effervescent Atomizer}

A full body transparent effervescent atomizer was designed and manufactured to enhance direct visualization of the two-phase mixture inside the atomizer. The atomizer main parts are liquid and gas inlets, mixing chamber, aerator tube, and the exit orifice, as shown schematically in Fig. 2a. The gas and liquid inlet ports were machined in a square cross section acrylic block with the dimensions of $51 \mathrm{~mm} \mathrm{x}$ $51 \mathrm{~mm} \times 31.8 \mathrm{~mm}$. The exit orifice with a diameter and length of $1.56 \mathrm{~mm}$ and $2.2 \mathrm{~mm}$ respectively was machined in a square cross section acrylic block with the dimensions of $51 \mathrm{~mm}$ x $51 \mathrm{~mm} \times 28.6 \mathrm{~mm}$. The two blocks which have the inlet ports and the exit orifice were connected together by an acrylic tube with $3 \mathrm{~mm}$ thickness, $9.76 \mathrm{~mm}$ inner diameter and $82.6 \mathrm{~mm}$ length, the lower part of this tube was referred as the mixing chamber of the atomizer. A convergent section of $90^{\circ}$ has machined upstream the exit orifice as a reduction section from the diameter of the mixing chamber to the exit orifice diameter. The aerator was made of $0.5 \mathrm{~mm}$ thick brass tube with inner and outer diameters of 3 $\mathrm{mm}$ and $4 \mathrm{~mm}$, respectively. The aerator tube has 18 holes in four columns which are $90^{\circ}$ apart. These columns were arranged in an alternative manner with 4 and 5 holes in each. The diameter of each hole is 0.5 $\mathrm{mm}$ and the distance between the holes in each column is $10 \mathrm{~mm}$. The atomizer was "inside-out" gas injection geometry whereby the gas flows inside the aerator tube 
Farid A. Hammad, Aly H. Gadallah, E. A. El-Shenawy, Zakarya A. Zyada " Experi.. "

(which placed inside the acrylic tube) and bubbles outward into the surrounding liquid.

To study the effect of the bubble size in the spray quality, two bubble breakers with the different design were installed in the mixing chamber for controlling the number and size of the bubbles. The two breakers were made from acrylic with outer diameter and length of $9.66 \mathrm{~mm}$ and $8 \mathrm{~mm}$, respectively. All the breakers design dimensions are as shown in the Fig.2b. Each breaker has 9 holes of $1 \mathrm{~mm}$ diameter and 4 circular grooves of $0.75 \mathrm{~mm}$ radius at the breaker perimeter. The first bubble breaker has a flat base which named "flat end BB", and the second has a conical base which named "cone end BB". The "cone end BB" has 4 horizontal holes of $1.5 \mathrm{~mm}$ diameter and at a distance of $1.94 \mathrm{~mm}$ from the breaker top surface, these holes are used as a connection for the groove and the neighbor hole in the same plane. These breakers were installed inside the mixing chamber at a distance of $56.6 \mathrm{~mm}$ apart from the exit orifice.

\subsection{Shadowgraphy}

\subsubsection{Setup and Data Acquisition}

The used image visualization technique is known as shadowgraphy, which consists of CCD digital camera (12-bit, $1280 \times 1024$ pixels, $8 \mathrm{HZ}$ frequency) as the detector and double pulsed Nd: YAG laser $(\lambda=532 \mathrm{~nm})$ as the back illumination source. The laser beam was diffused using a ground glass diffuser (DG10-1500-A, Thorlabs), engineered diffuser (ED1-C50-MD, Thorlabs) and fluorescent plate for enhancing the quality, reducing the light speckles and uniform illumination of the image background. For the internal flow, a micro lens (Nikon AF Micro Nikkor $60 \mathrm{~mm}$ $\mathrm{f} / 2.8 \mathrm{D}$ ) used with the camera for the visualization of the bubbles inside the transparent block of the exit orifice with a field of view of $20 \mathrm{~mm} \times 16 \mathrm{~mm}$, as shown in Fig. 3. For the near nozzle and total spray structure the same arrangement was used with a field of view of $34 \mathrm{~mm} \times 27 \mathrm{~mm}$ and $211 \mathrm{~mm} \times 170 \mathrm{~mm}$, respectively. For the spray cone angle measurement, the laser beam diffuser devices are replaced with -10 $\mathrm{mm}$ spherical lens which converts the laser beam to a thin sheet. The camera was perpendicular to the laser sheet which passes through the core of the spray and the exit orifice. For the droplet visualization, a magnification system as shown in Fig. 1b was connected to the camera; this system (i.e. the bellows plus the close-up lenses) enables us to have a wide range of fields of view (magnification degree) at a suitable focal distance. We found that $2.6 \mathrm{~mm} \times 2.1$ $\mathrm{mm}$ field of view was suitable for the spray droplet diameters at $155 \mathrm{~mm}$ downstream the exit orifice.

\subsubsection{Data processing}

The qualitative and quantitative characterization of the internal two-phase flow at every gas-to-liquid ratio (GLR) are carried out by analyzing the shadowgraphs which are obtained from the direct imaging of the internal flow inside the mixing chamber directly upstream the atomizer exit orifice, where the two-phase flow mixture should be fully developed. A set of 40 shadowgraphs of the internal flow is acquired at each GLR. The visual analysis of these shadowgraphs defines the internal flow regime. While the equivalent diameter of each bubble and subsequently the related statistics of the whole bubbles at each GLR is obtained by analyzing the shadowgraphs using an in-house algorithm, to reduce the error caused by the bubbles overlapping and interacting, this algorithm allowed the user to define three points at the perimeter of the bubbles, then the program detects them and computes their equivalent diameters. An average number of bubbles around $(250-350)$ is detected at each GLR. The quantitative characterization for the external flow (i.e. spray) is carried out by analyzing the shadowgraphs that are captured for the spray drops at a location of $155 \mathrm{~mm}$ directly downstream the exit orifice. A set of 200 shadowgraphs of spray drops is acquired at each GLR. These shadowgraphs are analyzed using an in-house algorithm in the Matlab environment, which detects each drop in the image and then computes its cross-sectional area and subsequently the equivalent area diameter. Hence, the drop size distributions, as well as the averaged diameters, are obtained, which are the spray quality indicators. An average number of drops around (1000-1200) is detected at each GLR. For the spray cone angle, a set of 50 images for the spray near the exit orifice is captured at each GLR and analyzed using commercial software 
Farid A. Hammad, Aly H. Gadallah, E. A. El-Shenawy, Zakarya A. Zyada " Experi.. "

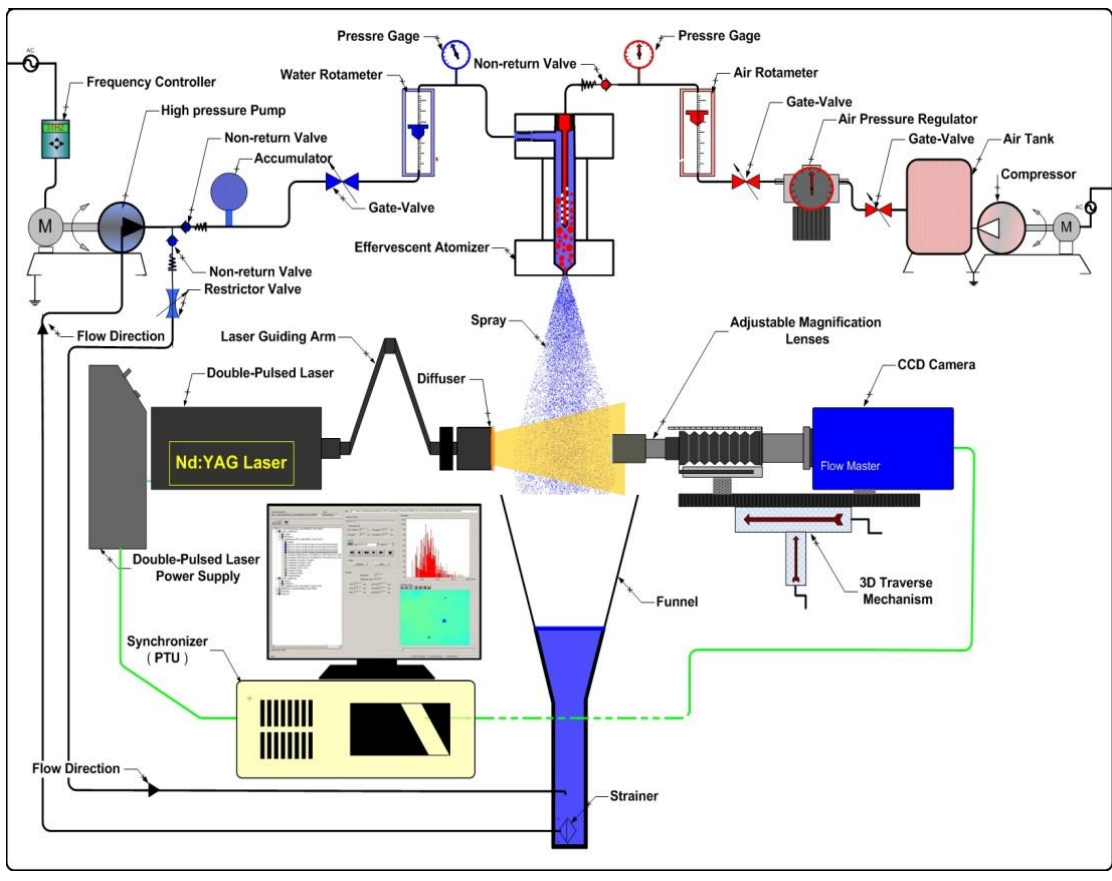

(a)

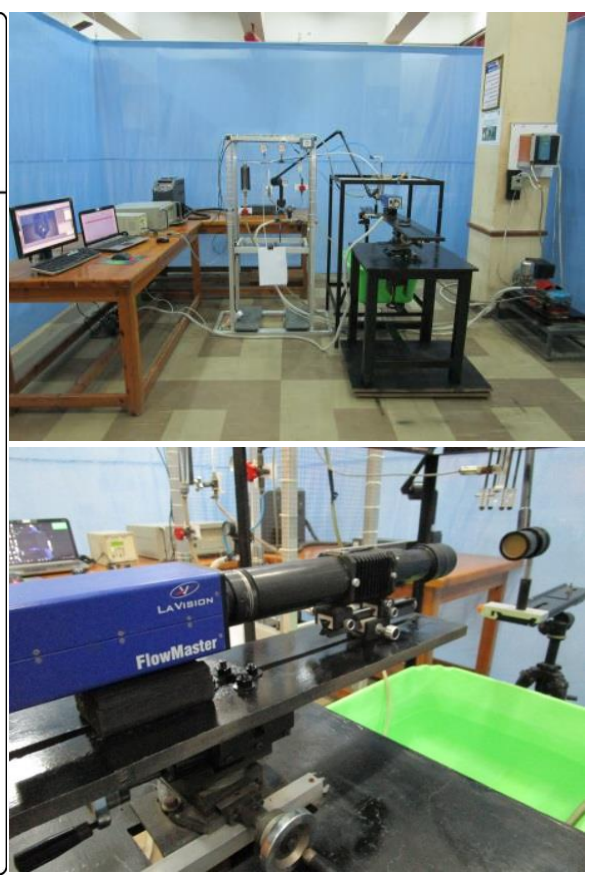

(b)

Fig.1: (a) Schematic drawing for the experimental rig with the droplet measurement and visualization system. (b) Photographs of the experimental rig and external flow (spray droplets) measurement and visualization system.

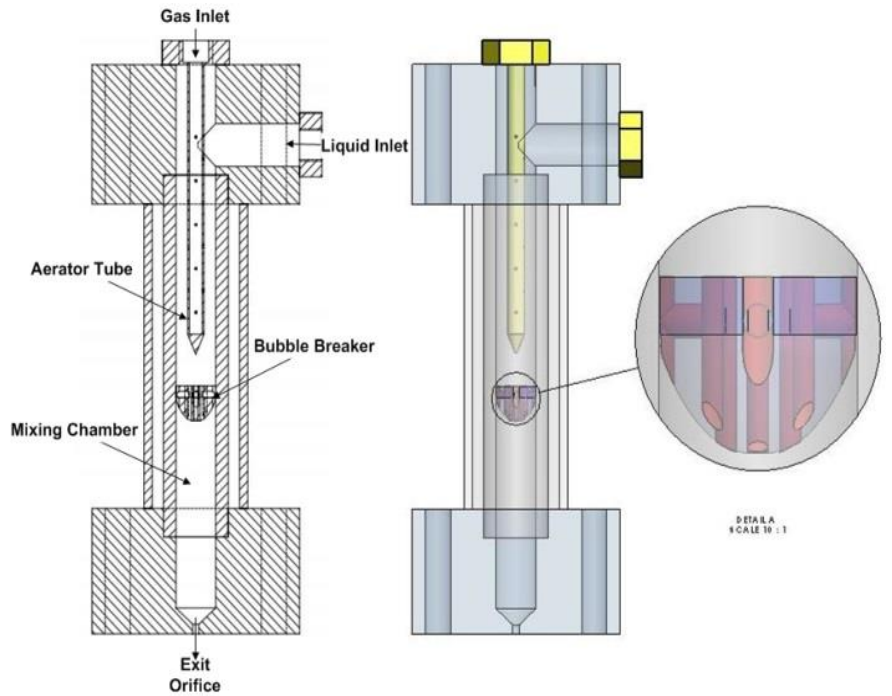

(a)

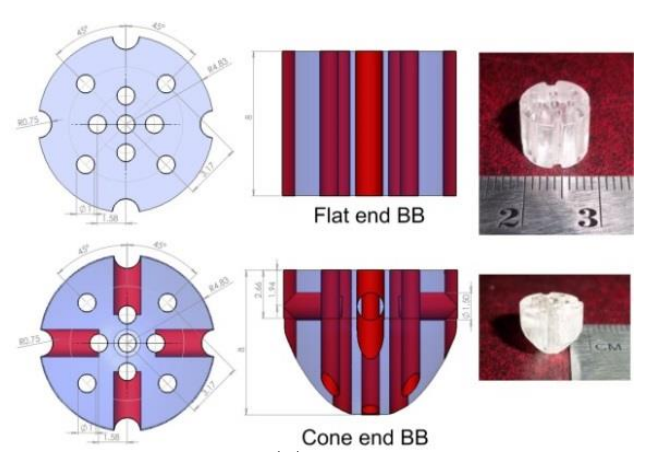

(b)

Fig. 2: (a) Scaled schematic for the atomizer with the cone end bubble breaker installed inside it and a cross section of it. (b) Scaled schematic with a photograph for the bubble breakers used in the study. 
Farid A. Hammad, Aly H. Gadallah, E. A. El-Shenawy, Zakarya A. Zyada " Experi.. "

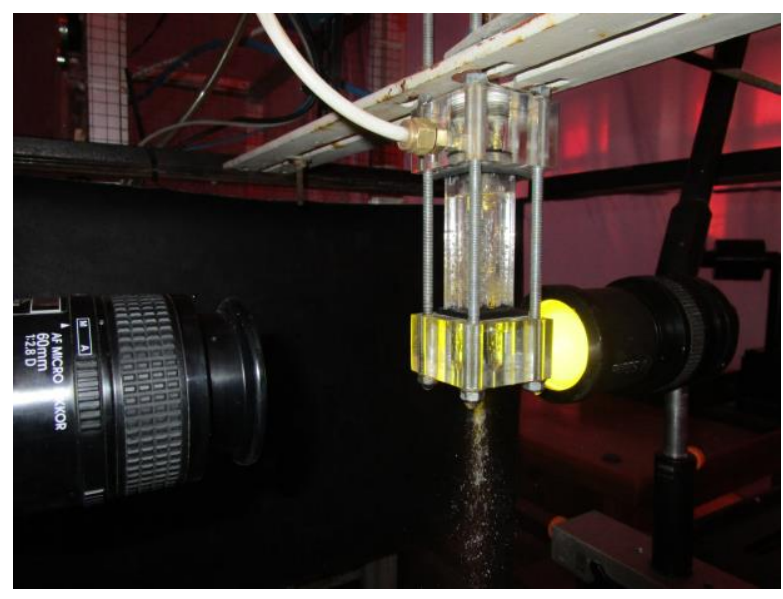

Fig.3. Photograph of the internal two phase flow measurement and visualization system.

(Geometry Package for DaVis 6.2 developed by LaVision, Germany). The output result from this program is the average spray cone angle at each GLR. By using the droplet and bubbles diameters, we can calculate some of the performance indicators such as the averaged diameters. Sauter mean diameter (SMD or D32), which is an important spray performance indicator, is a measure of the spray finest. It is a ratio between the total volume of the spray drops to the total surface area of the spray drops. So it is very important in the combustion applications as they need high droplets evaporation rates. The (SMD) is given by:

$$
D_{32}=\frac{\sum_{i=1}^{n} D_{i}^{3}}{\sum_{i=1}^{n} D_{i}^{2}} \ldots \ldots .(1)
$$

\section{RESULTS AND DISCUSSION}

The results in this section are presented in the following sequence: initially, investigating the type of the internal flow pattern is discussed, then the influence of the GLR as well as the installation of the bubble breaker inside the mixing chamber on some characteristic indicators of the internal two-phase flow.
Subsequently, the influence of the internal flow regime inside the mixing chamber (i.e. bubble size) on some of the produced spray characteristics will be discussed.

\subsection{Investigation the type of the internal two-phase flow pattern.}

The internal two-phase flow inside the atomizer is visualized and described using a digital camera. The visualization results for the two-phase flow show different two-phase flow patterns acquired under the atomizer operation with injection pressure $0.6 \mathrm{Mpa}$ and different GLRs of $0.71 \%, 1.29 \%, 1.73 \%, 2.02 \%$, $2.53 \%, 3.11 \%, 3.73 \%, 4.43 \%$ and $5.74 \%$. The actual patterns of the two-phase flow can be seen in Fig. 4. The observations are the following:

From Fig. 4a, the dominant feature at this GLR is formed of discrete groups of bubbles flow towards the exit orifice followed by slugs of liquid. There is relatively large bubble blank volumes in the mixing chamber, i.e. there is no homogeneous distribution for the gas-phase inside the mixing chamber and the convergent zone. Furthermore, a few numbers of slightly large bubbles is observed whereas the small bubbles, which have diameters slightly larger than, equal to, or less than the exit orifice diameter, are supernumerary. This is attributed to the low amount of the aerated gas-phase in the mixture, i.e. the law GLR. Since the external flow (i.e. spray) is relevant to the atomizer internal two-phase flow, wherefore the aforementioned internal flow regime leads to spray pulsation at the exit orifice due to the intermittent occurrence of single-phase and two-phase flow inside the exit orifice and the near nozzle spray structure for this case can be seen in Fig. 5. Subsequently, the observed flow pattern should be bubbly flow.

From Fig. $4 b-d$, by increasing the GLR, i.e. decrease the volume flow rate of the liquid and increasing the volume flow rate of the atomizing gas, the number of bubbles and slightly its size increase with a little deformation in its spherical shape, as well as the enhancement of its spreading and distribution in the mixing chamber. Also with increasing the GLR, increases the presence of elongated bubble series in the 
convergent zone, which decreases the intermittent two flow structures inside the exit orifice and subsequently the spray pulsation. Also, the observed flow pattern should be bubbly flow.

which is due to the small bubbles coalescence while aerating the liquid by large gas volume flow rate. Also, the width of the elongated bubbles in the convergent zone increases with increasing the GLR. The observed flow pattern should be plug-bubble to slug-bubble flow, this range of GLRs represents the unsteady transition stage between the bubble flow and annular flow.

From Fig. 4i, further increase in GLR, the gas phase becomes a column in the center of the mixing chamber with some discrete large bubbles on the wall of the mixing chamber. Subsequently, the observed flow should be bubble-annular flow.

\subsection{Influence of the bubble breakers on the internal} two-phase flow and its characteristic indicators.

\subsubsection{The qualitative analysis:}

After investigating the internal flow pattern in the (section 4.1) at the defined range of GLRs. In this section, we control this flow by using two bubble breakers which are installed inside the mixing chamber. They are used for the fragmentation of the large bubble or gas slugs and convert it to smaller ones. Novel design for breakers (flat end $\mathrm{BB}$ and cone end $\mathrm{BB}$ ) are used for this objective.

The shadowgraphs in Fig. 6. Shows the two-phase flow inside the mixing chamber downstream the breakers by a distance of $30 \mathrm{~mm}$ at different GLRs.

For the GLR $=0.77 \%$ in Fig. 6, the shadowgraphs $(a, b)$ shows a lot of small bubbles that distinctly have diameters smaller than the exit orifice diameter. Also, these bubbles seem to have the same size or it nearly have a narrow size distribution. Furthermore, these bubbles have a quasi-homogenous distribution in the flow; this is due to the low amount of the atomizing gas which is aerated in the liquid (i.e. low GLR). Finally, the flow seems to be fully developed. We must note that the flow pattern which exists upstream the flat end $\mathrm{BB}$ and the cone end $\mathrm{BB}$ is nearly the same as the flow pattern that in Fig. 4a (the case of without BB), whereas Fig. 6a and b shows the flow pattern (bubbly flow) downstream the flat and the cone end BBs,
From Fig. 4e-h, with a continual increase in GLR, the volume of bubbles begins to increase largely relative to the diameter of the mixing chamber. Furthermore, decreasing in the small bubbles number respectively. This gives clear evidence that flat and the cone end BBs have a great ability to convert the big bubbles to smaller ones with homogenous distribution in the flow.

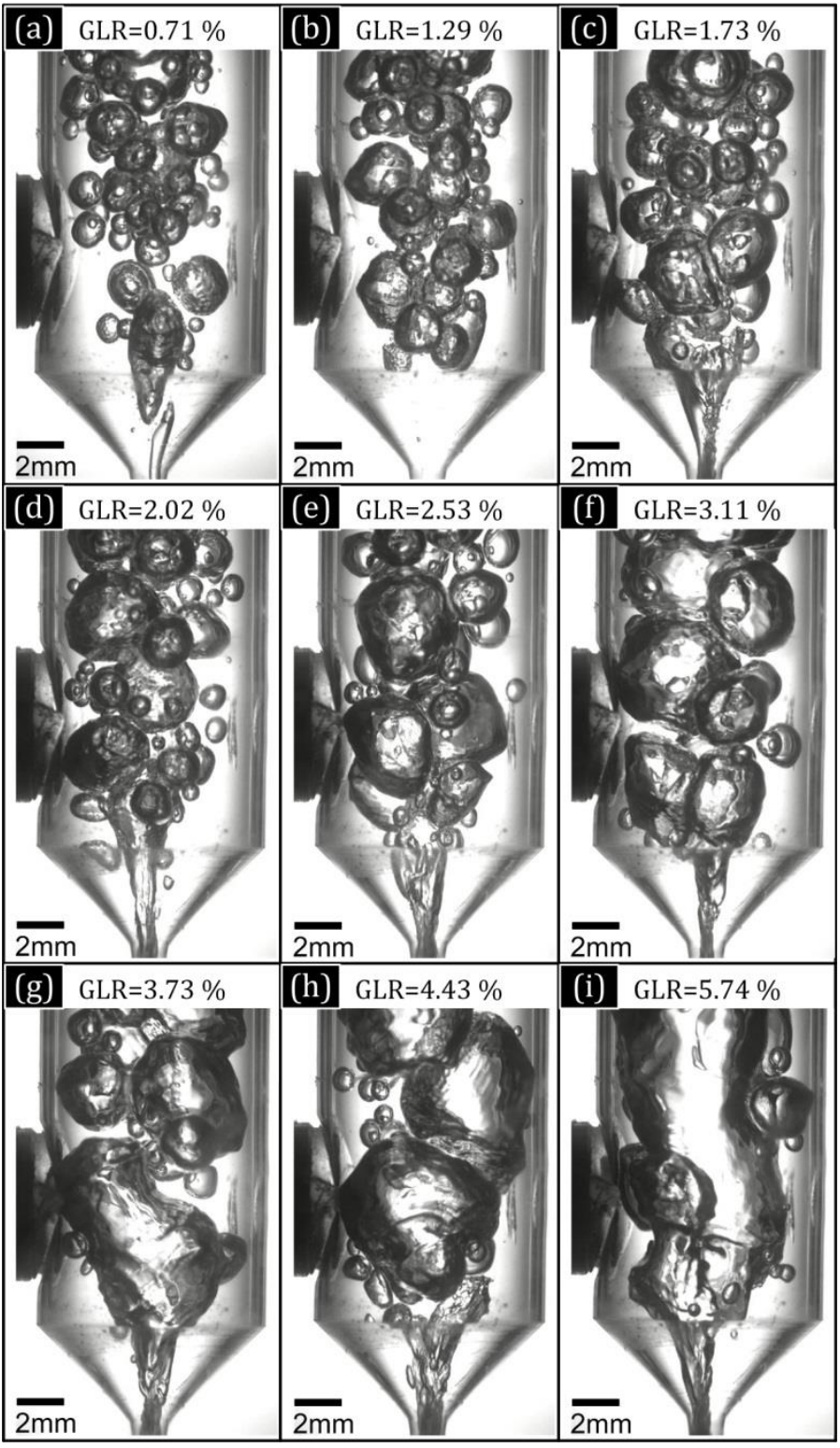

Fig. 4. Visualization of two-phase flow inside atomizer mixing chamber at various GLRs, $(\mathrm{Pg}=0.6 \mathrm{Mpa})$ 
Farid A. Hammad, Aly H. Gadallah, E. A. El-Shenawy, Zakarya A. Zyada " Experi.. "

For the GLR $=1.21 \%$ in Fig. 6, the flow pattern which exists upstream the flat end $\mathrm{BB}$ and the cone end $\mathrm{BB}$ is nearly the same as the flow pattern that is in the Fig. $4 \mathrm{~b}$ (the case of without BB), whereas Fig. $6 \mathrm{c}$ and d shows the flow pattern (bubbly flow) downstream the flat and the cone end BBs, respectively. Increasing the GLR slightly increases the diameter of fragmented bubbles sizes as well as enhances its distribution in the mixing chamber. There is no notable difference in the

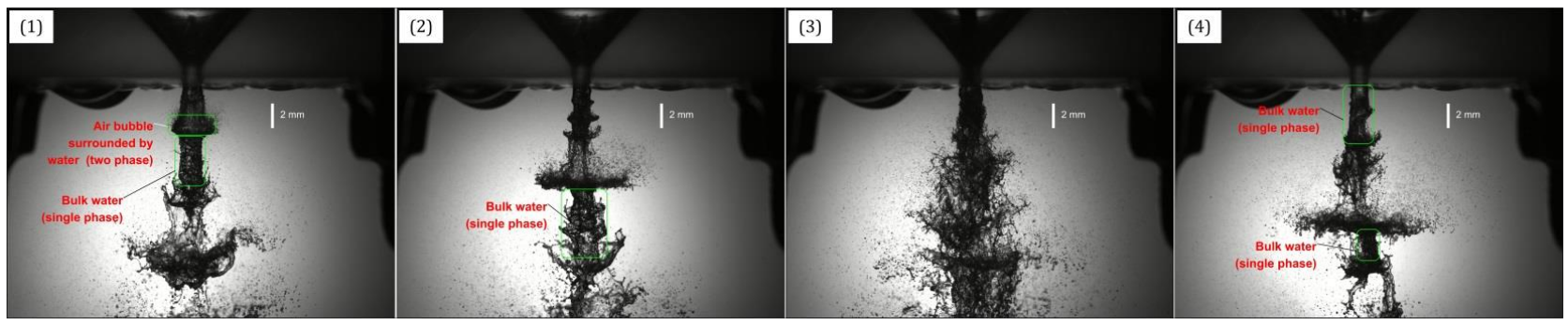

Fig. 5. Consecutive near nozzle spray structure shadowgraphs at $\mathrm{GLR}=0.71 \%,(\mathrm{Pg}=0.6 \mathrm{Mpa})$.

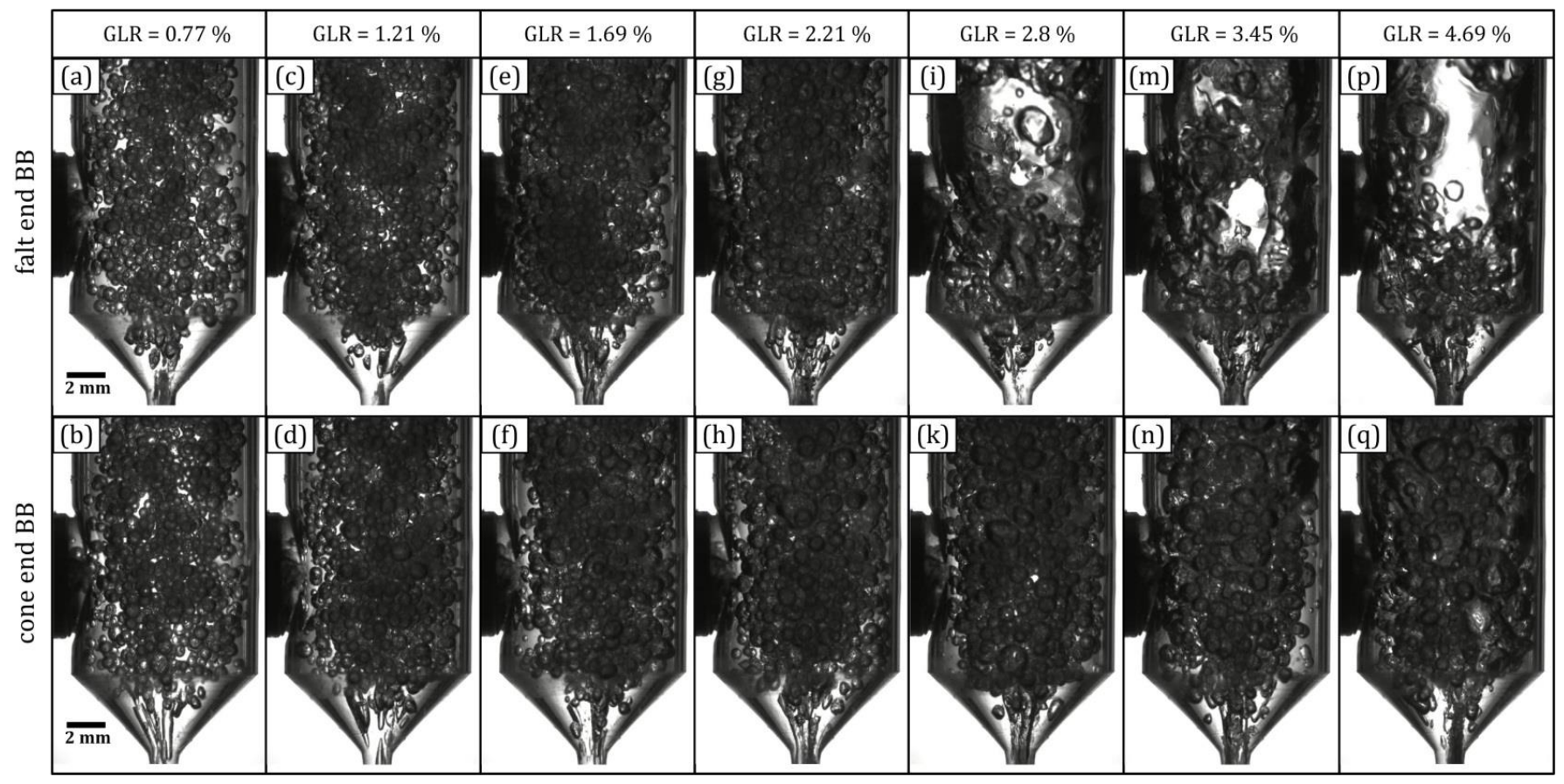

Fig. 6. Visualization of two-phase flow inside the mixing chamber downstream the BB at various GLRs, $(\mathrm{Pg}=0.6$ Mpa).

bubble sizes between that in Fig. 6c and d, namely, the two bubble breaker nearly do the same function at this GLR. The only difference is in the bubbles distribution in the mixing chamber which is slightly more homogeneous in the case of cone end BB (i.e. Fig. 6d) than that in the case of flat end BB (i.e. Fig. 6c).

For the GLR $=1.69 \%$ in Fig. 6, the flow pattern which exists upstream the flat end BB and the cone end 
BB slightly differs from the flow pattern that is in the Fig. 4c (the case of without BB), whereas Fig. 6e and $f$ shows the flow pattern (bubbly flow) downstream the flat and the cone end BBs, respectively. Again, with increasing the GLR, the fragmented bubbles size downstream the bubble breakers increase. Also, we can note in the Fig. $6 f$ that the bubbles don't have the same sizes. Some bubbles have sizes larger than the majority comparing to that in the Fig. $6 \mathrm{e}$.

numbers continually increase with increasing the GLR. Once more, there are some large bubbles relative to the others in the Fig. 6 h, whereas the bubbles in the Fig. $6 \mathrm{~g}$ are more homogenous in sizes comparing to Fig. $6 \mathrm{~h}$.

For the GLR $=2.8 \%$ in Fig. 6, the flow pattern which exists upstream the flat end $\mathrm{BB}$ and the cone end $\mathrm{BB}$ is medium between the flow patterns that are in the Fig. $4 \mathrm{e}$ and $\mathrm{f}$ (the case of without BB), whereas Fig. $6 \mathrm{i}$ and $\mathrm{k}$ shows the flow pattern downstream the flat and the cone end BBs, respectively. Fig. 6i shows a gas slug in the upper half of the shadowgraph followed by bubbly flow in the under half. On the other hand, for the cone end BB, the shadowgraph in Fig. 6k shows a bubbly flow.

For the GLR $=3.45 \%$ in Fig. 6, the flow pattern which exists upstream the flat end $\mathrm{BB}$ and the cone end $\mathrm{BB}$ is medium between the flow patterns that are in the Fig. $4 \mathrm{f}$ and $\mathrm{g}$ (the case of without BB), whereas Fig. $6 \mathrm{~m}$ and $\mathrm{n}$ shows the flow pattern downstream the flat and the cone end BBs, respectively. The flow pattern in the shadowgraph Fig. $6 \mathrm{~m}$ nearly is the same as that in the Fig. 6i. While the bubbles size in the Fig. $6 n$ increases with increasing the GLR.

Finally, for the GLR $=4.69 \%$ in Fig. 6, the flow pattern which exists upstream the flat end $\mathrm{BB}$ and the cone end $\mathrm{BB}$ is near to the flow pattern that in Fig. $4 \mathrm{~h}$ than that in the Fig. 4i (the case of without BB), whereas Fig. $6 p$ and $q$ shows the flow pattern downstream the flat and the cone end BBs, respectively. The shadowgraph in Fig. $6 p$ shows that the upper three quarters of the figure consists of a large gas slug, while the lower quarter consists of separate bubbles. This shadowgraph in Fig. 6p reveals that, with increase the GLR, the gas slug size downstream the breaker increases. Likewise, the bubbles sizes in the bubbly flow presented in the Fig. $6 q$ increase with the increment of the GLR.
For the GLR $=2.21 \%$ in Fig. 6, the flow pattern which exists upstream the flat end $\mathrm{BB}$ and the cone end $\mathrm{BB}$ is medium between the flow patterns that are in the Fig. $4 \mathrm{~d}$ and e (the case of without BB), whereas Fig. $6 \mathrm{~g}$ and $h$ shows the flow pattern (bubbly flow) downstream the flat and the cone end BBs, respectively. The fragmented bubbles sizes and

\subsubsection{The quantitative analysis:}

In this section, the quantitative analyses of the influence of the bubble breakers on the internal twophase flow are investigated. Fig. 7 shows the results for the bubble SMD (D32) inside the mixing chamber directly upstream the exit orifice respectively with the change in the GLR, while the atomizer operating with and without the bubble breakers. First, for all cases,

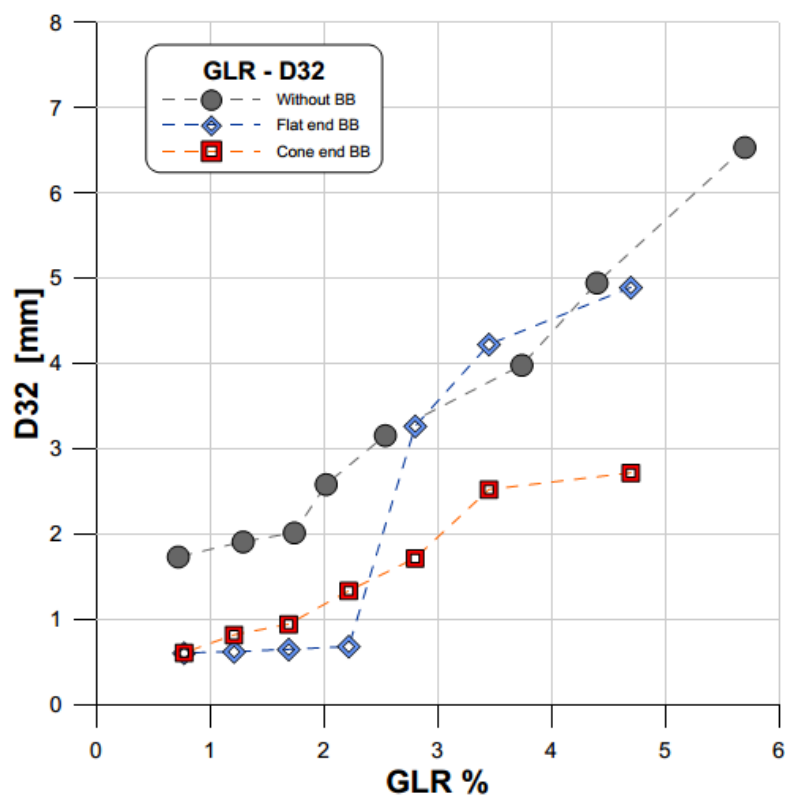

Fig.7. Bubbles SMD (D32) versus GLR, $(\mathrm{Pg}=$ $0.6 \mathrm{Mpa})$.

increasing the GLR leads to increase the bubbles SMD. The largest SMD is $6.54 \mathrm{~mm}$ for the case of without $\mathrm{BB}$, while the smallest SMD is $0.605 \mathrm{~mm}$ for the case of the flat end BB. Also, the figure shows that the decrement percentage in the SMD for the fragmented bubbles through the flat end BB at GLRs of $0.77 \%$, $1.21 \%, 1.69 \%, 2.21 \%, 2.8 \%$, and $4.69 \%$ are $66 \%$, $67.29 \%, 67.63 \%, 75.63 \%, 2.49 \%$, and $7.65 \%$, respectively, relative to the case of without $\mathrm{BB}$. While the decrement percentage in the SMD for the 
Farid A. Hammad, Aly H. Gadallah, E. A. El-Shenawy, Zakarya A. Zyada " Experi.. "

fragmented bubbles through the cone end $\mathrm{BB}$ at the same GLRs are $65.81 \%, 56.96 \%, 52.84 \%, 52.32 \%$, $48.75 \%$, and $48.71 \%$, respectively. At the GLR of $3.45 \%$, the SMD for the fragmented bubbles through the flat end BB increases by $11.51 \%$, while the SMD for the fragmented bubbles through the cone end $\mathrm{BB}$ decreases by $33.41 \%$ all relative to the case without BB. It is found that, the SMD for the fragmented bubbles through the flat end BB at GLRs of $0.77 \%$, $1.21 \%, 1.69 \%, 2.21 \%$ are $0.56 \%, 23.99 \%, 31.36 \%$, and $48.89 \%$ smaller than the SMD for the fragmented bubbles through the cone end $\mathrm{BB}$, respectively. At the GLRs of $2.8 \%, 3.45 \%$, and $4.69 \%$, the SMD for the fragmented bubbles through the flat end BB are $90.27 \%, 67.46 \%$, and $80.1 \%$ larger than the SMD for the fragmented bubbles through the cone end BB, respectively. So, we can conclude that:

- The cone end BB works very well (have good fragmentation process) at all the operating GLRs relative to the case of without $\mathrm{BB}$.

- The flat end BB works very well only at the GLRs $\leq$ $2.21 \%$ relative to the case of without BB (by large decrement percentage), as well as, relative to the case of the cone end BB (by small decrement percentage).

- The flat end BB have bad fragmentation process at the GLRs $>2.21 \%$ relative to the case of without BB (by small decrement percentage), as well as, relative to the case of the cone end BB (by large increment percentage).

- The largest decrement percentage in the fragmented bubbles SMD is $75.63 \%$ at GLR of $2.21 \%$ for the case of the flat end $\mathrm{BB}$ relative to the case of without BB.

3.3. Influence of the internal two-phase flow (i.e. bubble breakers) on the external flow (i.e. spray) characteristics.

In this section, the quantitative analyses of the influence of the internal flow regime as well as the bubble breakers on the spray droplet size and the spray cone angle.

\subsubsection{Spray droplet size:}

Fig. 8 shows the results for the droplet SMD (D32) outside and downstream the exit orifice with the change in the GLR, while the atomizer operating with and without the bubble breakers. First, for the case of without $\mathrm{BB}$ and for the cone end $\mathrm{BB}$, the droplet SMD decreases with increasing the GLR. While for the case of the flat end BB, with increasing the GLR, the droplet SMD decreases till the GLR of $2.21 \%$, and a further increase in the GLR leads to increase the droplet SMD. The largest SMD is $1.21 \mathrm{~mm}$ for the case of without BB at the GLR of $0.71 \%$, while the smallest SMD is 0.108 $\mathrm{mm}$ for the case of the cone end BB at the GLR of $4.69 \%$. Also, the figure shows that the decrement percentage in the droplet SMD for the case of the flat end BB at GLRs of $0.77 \%, 1.21 \%, 1.69 \%, 2.21 \%$, and $2.8 \%$ are $68.74 \%, 64.7 \%, 62.03 \%, 59.91 \%$, and 14.65 , respectively, relative to the case of without $\mathrm{BB}$, while

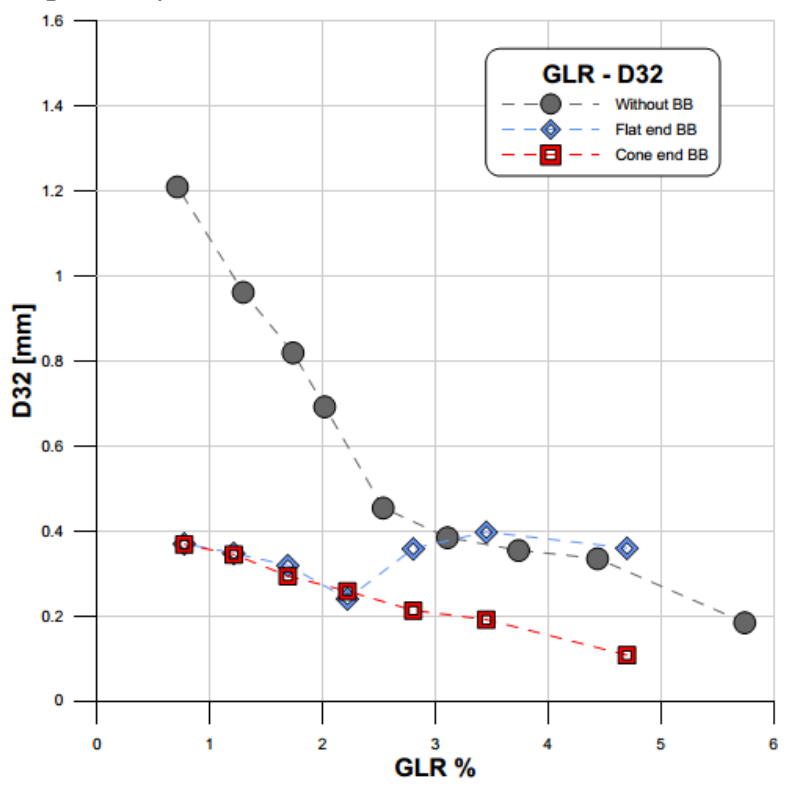

Fig.8. Droplet SMD (D32) versus GLR. (Pg= 0.6 Mpa)

at GLRs of $3.45 \%$ and $4.69 \%$ the droplet SMD increases by $8.91 \%$ and $19.9 \%$, respectively, also relative to the case of without BB. For the case of the cone end $\mathrm{BB}$, the decrement percentage in the droplet SMD at the GLRs of $0.77 \%, 1.21 \%, 1.69 \%, 2.21 \%$, $2.8 \%, 3.45 \%$, and $4.69 \%$ are $68.88 \%, 65.01 \%, 65 \%$, $56.95 \%, 49.27 \%, 47.59 \%$, and $64.02 \%$, respectively, relative to the case of without $\mathrm{BB}$. There is no notable difference in the droplet SMD for the cases of the flat and the cone end BBs at the GLRs of $0.77 \%, 1.21 \%$, $1.69 \%$, and $2.21 \%$, while the droplet SMD for the case of the cone end BB at GLRs of 2.8\%, 3.45\% and $4.69 \%$ 
are $40.57 \%, 51.88 \%$, and $69.99 \%$ smaller than the droplet SMD for the case of the flat end BB. This notable difference is due to the non-homogeneous internal two-phase flow regime inside the mixing chamber downstream the flat end BB (at the last three GLRs) relative to the quasi- homogeneous internal flow regime in the case of the cone end $\mathrm{BB}$, and this gives an advantage to the cone end BB design. Finally, the largest decrement percentage in the droplet SMD is $68.88 \%$ at GLR of $0.77 \%$ for the case of the cone end $\mathrm{BB}$ relative to the case of without BB. From the Fig.7 and 8 , we can conclude that the structure of the internal two-phase flow upstream the atomizer exit orifice at any given GLR has a strong effect on the droplet SMD of the produced spray. Overall, at any given GLR, decreasing the bubbles size inside the mixing chamber leads to decrease the droplet SMD of the produced spray.

\subsubsection{Spray cone angle:}

Fig. 9 shows the results for the spray cone angle downstream the exit orifice with the change in the GLR, while the atomizer operating with and without the bubble breakers. First, the trend for all cases shows that the spray cone angle increases with increasing the GLR, this is attributed to increasing the gas phase which increases the disruptive forces acting on the liquid. The difference in the trend fitting between the two cases of bubble breakers is small, whereas they have a large difference relative to the case of without BB. At any given GLR, the produced spray cone angle for the cases of flat and cone end BBs is larger than that for the case of without $\mathrm{BB}$, this can be attributed to decreasing the bubbles sizes inside the mixing chamber as well as the bubbles have a quasi-homogenous distribution in the flow. The largest spray cone angle is 41.7 for the case of the cone end BB at the GLR of $3.45 \%$, while the smallest spray cone angle is 22.3 for the case of without BB at the GLR of $0.72 \%$. The largest increment percentage in the spray cone angle is $69.97 \%$ at GLR of $0.77 \%$ for the case of the cone end BB relative to the case of without BB. Finally, the bestproduced spray cone angles are for the case of the cone end $\mathrm{BB}$ relative to the other two cases.

\section{CONCLUSIONS}

An experimental study was conducted to investigate the effect of the internal two-phase flow pattern as well as the bubbles sizes inside the atomizer on some characteristic indicators of the produced spray. From the investigation of the internal two-phase flow pattern inside the atomizer, the flow is found to be bubbly flow at GLRs $\leq 1.73 \%$ to a bubble-annular flow at the GLR of $(5.74 \%)$, passing through an unsteady transition stage between these two patterns. Novel bubble breakers were designed and used to control the bubbles sizes. The results show that the bubble breakers efficiently fragment large bubbles to smaller ones. Overall, the cone end $\mathrm{BB}$ design is found to be more efficient in the bubbles fragmentation and

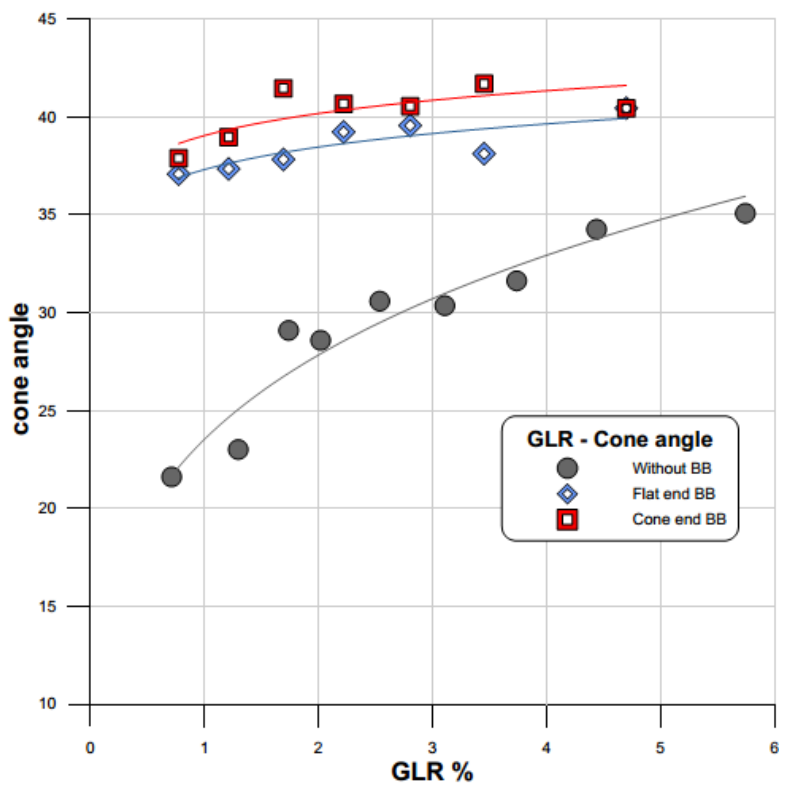

Fig.9. Spray cone angle versus GLR, $(\mathrm{Pg}=0.6 \mathrm{Mpa})$

producing a homogeneous bubbly flow than the flat end $\mathrm{BB}$ design. The results show that increasing the gas to liquid ratio leads to decrease the spray droplet SMD while increases the produced spray cone angle. Also, at a given GLR, decreasing the bubbles sizes inside the atomizer improves the quality of the produced effervescent spray in the form of decreasing the droplet SMD and increasing the spray cone angle. Finally, the results demonstrate that the characteristics of the produced spray by the atomizer with the presence of the cone end $\mathrm{BB}$ are the best relative to the other two 
cases.

\section{REFERENCES}

[1] Khan, M. A., \& Ghouri, A. M., "Environmental Pollution: Its Effects on Life and Its Remedies," Res. World J. Arts, Sci. Commer., vol. 2, no. 2, pp. 276-285, 2011.

[2] Martínez, I. , "Environmental effects and hazards in combustion," http://webserver.dmt.upm.es/ isidoro/. pp. 1-16, 2015.

[3] Sovani, S. D., Sojka, P. E., \& Lefebvre, A. H., "Effervescent atomization," Prog. Energy Combust. Sci., vol. 27, no. 4, pp. 483-521, 2001.

[4] Gadgil, H. P., \& Raghunandan, B. N. , "Some features of spray breakup in effervescent atomizers," Exp. Fluids, vol. 50, no. 2, pp. 329338, 2011.

[5] Ballal, A. H. L. D. R., \& Lefebvre, A. , Gas Turbine Combustion Alternative Fuels and Emissions. (Taylor and Francis Group, LLC), 2010.

[6] Lipp C.W, "Sprays," in Kirk-Othmer Encyclopedia of Chemical Technology, vol. 23, (John Wiley \& Sons, Inc), pp. 1-32, 2006.

[7] Fritsching, U., "Spray Systems," in Multiphase Flow Handbook, (Taylor \& Francis Group, LLC), pp. 1-100, 2006.

[8] Ashgriz, N., Handbook of atomization and sprays: theory and applications. Springer Science \& Business Media, 2011.

[9] Gomez, J., Fleck, B., Olfert, J., \& McMillan, J., "Characterization of a horizontal two-phase spray from an effervescent atomizer," Proc. ILASSEurope, 23rd Annu. Conf. Liq. At. Spray Syst., pp. 1-9, 2010.

[10] Jobehdar, M. H., Gadallah, A. H., Siddiqui, K., \& Chishty, W. A. , "Investigation of Two-Phase Flow in an Effervescent Atomizer," ASME 2014 4th Jt. US-European Fluids Eng. Div. Summer Meet. collocated with ASME 2014 12th Int. Conf. Nanochannels, Microchannels, Minichannels . Am. Soc. Mech. Eng., pp. 1-7, 2014.

[11] Lefebvre, A. H., Wang, X. F., \& Martin, C. A. , "Spray characteristics of aerated-liquid pressure atomizers," J. Propuls. Power, vol. 4, no. 4, pp. 293-298, 1988.

[12] Lefebvre, A. H. , "A novel method of atomization with potential gas turbine applications.," Def. Sci. J., vol. 38, no. 4, pp. 353-362, 1988.

[13] Roesler, T. C., \& Lefebvre, A. H., "Studies on aerated-liquid atomization," Int. J. Turbo Jet
Engines, vol. 6, no. (3-4), pp. 221-230, 1989.

[14] Wang, X. F., Chin, J. S., \& Lefebvre, A. H., "Influence of gas-injector geometry on atomization performance of aerated-liquid nozzles," Int. J. Turbo Jet Engines, vol. 6, no. 34, pp. 271-280, 1989.

[15] Lawler, A., Wade, R. A., Sojka, P. E., \& Gore, J. P., "Flame length and pollutant emission characteristics of effervescent atomizer/burner stabilized jet flames, combustion fundamentals and applications," Proc. Tech. Meet. Cent. States Sect. Combust. Inst., 1996.

[16] Satapathy, M. R., Sovani, S. D., Sojka, P. E., Gore, J. P., Eckerle, W. A., \& Crofts, J. D., "The effect of ambient density on the performance of an effervescent atomizer operating in the $\mathrm{MPa}$ injection pressure range," Proc. Tech. Meet. Cent. States Sect. Combust. Inst., pp. 76-80, 1998.

[17] Wade, R. A., Sojka, P. E., \& Gore, J. P., "Effervescent atomization using high supply pressures," Proc. 9th Annu. Conf. Liq. At. Spray Syst. San Fr. CA, pp. 263-70, 1996.

[18] Subramanian, V., \& Sankar, A., "Swirl effervescent atomizer for spray combustion," Proc. ASME Heat Transf. Div., vol. 37, no. 2, pp. 18-22, 1995.

[19] Loebker, D. W., \& Empie Jr, H. L., "High Mass Flowrate Effervescent Spraying of a High Viscosity Newtonian Liquid," 10th Annu. Conf. Liq. At. Spray Syst. (ILASS Am. '97), Ottawa, pp. 253-260, 1997.

[20] Hájek, J., Dohnal, M., Vondál, J., \& Broukal, J., "Analysis of effervescent spray quality for oilfired furnace application," Clean Technol. Environ. Policy, vol. 17, no. 5, pp. 1195-1205, 2015.

[21] Stähle, P., Gaukel, V., \& Schuchmann, H. P. , "Investigation on the Applicability of the Effervescent Atomizer in Spray Drying of Foods: Influence of Liquid Viscosity on Nozzle Internal Two-Phase Flow and Spray Characteristics," J. Food Process Eng., vol. 38, no. 5, pp. 474-487, 2015.

[22] Schröder, J., Kraus, S., Rocha, B. B., Gaukel, V., \& Schuchmann, H. P., "Characterization of gelatinized corn starch suspensions and resulting drop size distributions after effervescent atomization," J. Food Eng., vol. 105, no. 4, pp. 656-662, 2011.

[23] Broniarz-Press, L., Ochowiak, M., \& Woziwodzki, S., "Atomization of PEO aqueous solutions in effervescent atomizers," Int. J. Heat Fluid Flow, vol. 31, no. 4, pp. 651-658, 2010.

[24] Song, S. H., \& Lee, S. Y., "Study of atomization 
Farid A. Hammad, Aly H. Gadallah, E. A. El-Shenawy, Zakarya A. Zyada " Experi.. "

mechanism of gas/liquid mixtures flowing through Y-Jet atomizers," At. sprays, vol. 6, no. 2, pp. 193-209, 1996.

[25] Lin, K. C., Kennedy, P. J., \& Jackson, T., "Structures of internal flow and the corresponding spray for aerated-liquid injectors," in AIAA/ASME/SAE/ASEE Joint Propulsion Conference and Exhibit, 37 th, Salt Lake City, UT, 2001.

[26] Lörcher, M., Schmidt, F., \& Mewes, D., "Flow field and phase distribution inside effervescent atomizers," 9th ICLASS, Sorrento, Italy, 2003.

[27] Jedelsky, J., \& Jicha, M. , "Effervescent atomizertemporal and spatial variations of spray structure," Proc. 10th Int. Congr. Liq. At. Spray Syst. ICLASS, pp. 1-8, 2006.

[28] Liu, M., Duan, Y., \& Zhang, T., "Evaluation of effervescent atomizer internal design on the spray unsteadiness using a phase/Doppler particle analyzer," Exp. Therm. Fluid Sci., vol. 34, no. 6, pp. 657-665, 2010.
[29] Mlkvik, M., Stähle, P., Schuchmann, H. P., Gaukel, V., Jedelsky, J., \& Jicha, M., "Twin-fluid atomization of viscous liquids: The effect of atomizer construction on breakup process, spray stability and droplet size," Int. J. Multiph. Flow, vol. 77, pp. 19-31, 2015.

[30] Huang, X., Wang, X., \& Liao, G., "Visualization of two phase flow inside an effervescent atomizer," J. Vis., vol. 11, no. 4, pp. 299-308, 2008.

[31] Sutherland, J. J., Sojka, P. E., \& Plesniak, M. W., "Entrainment by ligament-controlled effervescent atomizer-produced sprays," Int. J. Multiph. Flow, vol. 23, no. 5, pp. 865-884, 1997.

[32] Jagannathan, T. K., Nagarajan, R., \& Ramamurthi, K., "Effect of ultrasound on bubble breakup within the mixing chamber of an effervescent atomizer," Chem. Eng. Process. Process Intensif., vol. 50, no. 3, pp. 305-315, 2011. 